\title{
The Relationship Between Parent Attachment and Behavior Problems of Adolescences During the Epidemic of COVID-19: The Mediating Role of Resilience
}

\author{
Zhu Zhuyue \\ ${ }^{1}$ Guangdong Experimental High School \\ ${ }^{1}$ Corresponding author. Email: 2083594293@qq.com
}

\begin{abstract}
Objective: The incidence of behavior problems is very high among adolescents. Previous studies have shown that behavior problems can affect the physical and mental health of adolescents. And behavior problems closely relates to adolescences' academic achievement, interpersonal communication, and personality development. While in 2020, the influence of COVID-19 upon the mental health of the public has received great concern. As a public pandemic, whether it could increase the incidences of behavior problem of adolescents is still unknown. Considering the protective factors of behavior problems, existing studies have found that family-related variables are very important protective factors for problem behaviors. However, whether and how family-related factors could influence behavior problems during this special time is unclear. From the view of positive psychology and attachment theory, the current study investigated the characteristics of behavior problems of adolescence and the influence of parent attachment as well as the mediating role of resilience during the epidemic of COVID-19.

Methods: Sixty-nine adolescents aged 12-18 in Jiangxi, Guangdong, and Hebei provinces of China were recruited during the epidemic of COVID-19as participants $\left(\mathrm{M}_{\mathrm{age}}=15.83\right.$ years old, $\mathrm{SD}_{\text {age }}=1.06$ years old, including 19 boys $)$. They were asked to complete a series of online questionnaires including the Parent Attachment Scale, Strengths and Difficulties Questionnaire, Adolescent Resilience Scale, and some basic information scale.

Results: The results showed that during the epidemic of COVID-19: (1) adolescents' behavior problems were prominent, among which hyperactivity, attention problems, and emotional problems were significantly higher than other behavior problems; (2) among the relationship between parent-child attachment and adolescent problem behavior, the relationship between mother's trust and behavior problems, parents' alienation and behavior problems, and resilience and behavior problem were significant; and (3) the mediating analysis revealed that resilience plays a mediating role between mother's trust, father's alienation, mother's alienation, and behavior problem.

Conclusion: The results showed that attention problems and emotional problems were the main behavior problems of adolescents during the epidemic period of the COVID-19. During this time, parent attachment could act as a protective factor which relates to adolescents' behavior problem. What's more, parent attachment could predict adolescents' behavior problem through resilience. These results provide some implications for the intervention of problem behaviors during the COVID-19.
\end{abstract}

Keywords: adolescents, parent attachment, behavior problem, resilience, COVID-19

\section{INTRODUCTION}

Adolescence is an important stage for individual's physical and mental development, like the learning of knowledge or formation of outlook on life, world, and value. During this time, if the mental development is not mature enough, teenagers often can't correctly distinguish the right and wrong of the behaviors around them, which leads them to blindly pursue bad behavior. This will lead to teenagers' behavior problem. Teenagers' problem behavior is the behavior that violates the social norms in 
the process of socialization, resulting in adverse effects or even harmful for the society, others, or themselves[1]. Problem behaviors are divided into external behavior problems, internal behavior problems, and other types. external behavior problems are mainly manifested in individuals' negative adaptation to the external environment, such as hyperactivity, aggression, and discipline violation; Internal behavior problems are mainly manifested in the negative emotional feelings or internal experience, such as anxiety, depression, uneasiness, and withdrawal; Other types mainly include fault type, bad morality type, aggressive withdrawal type, social type, non-social type, self-evaluation, degeneration, neuroticism, learning ability, and living habits[2]. Problem behaviors of teenagers deviate from the normal standards in the process of socialization, they are obviously inconsistent with the normal behaviors of teenagers at their age. These behaviors also deviate from the requirements and behavior norms of family, school, and society, are not only detrimental to the development of personality and mental health, but also affect the current academic and interpersonal relationships of teenagers, even lead to criminal[1], [2]. Previous research shown that the detection rate of problem behavior among Chinese adolescents ranging from 13.7\% to $19.1 \%[3]$.

Previous research on teenagers' problem behavior shows that family factors are important influencing factors of teenagers' problem behavior[4]. According to the family ecosystem theory, the family can be regarded as a social system with a complex structure. It is composed of interrelated parts, in which each part will contribute to the overall function. Family is a stable system. The tangible and intangible rules produced by the interaction of family members constitute a relatively stable family structure, and a specific communication mode is formed among members. It can be seen that the generation of adolescent problem behavior is often closely related to the operation of the whole family system [5]. Studies have found that family structure could influence the outbreak of problem behaviors in adolescents [6]. In addition to the family ecosystem theory, Brownian Brenner's ecosystem theory also has similar expressions. The family ecosystem theory points out that in the environment factors which affecting individual physical and mental health, the innermost level of the structure is the micro system. Micro system refers to the direct environment of individual's activities and communication, which is constantly changing and developing. The second environmental level is the intermediate system, which refers to the connection or mutual relationship between micro systems, such as families, hospitals, and schools. The third environmental level is the outer system, which refers to those systems that are not directly involved but have an impact on their development, such as family friends, the media, and legal services. The fourth environmental system is the macro system, which refers to the extensive ideology, culture, and social stratum existing in the above three systems[5]. For teenagers, family is the most important meso environment in addition to school, and its impact on physical and mental health of teenagers cannot be ignored.

During the epidemic of COVID-19, the influence of family has been amplified as the life and the way of learning among adolescents has great change. Most of their time and interaction has been spent at home or online in the internet. Teenagers are in the stage of strong demand for interpersonal communication, their demand for social information has increased sharply. The outbreak of the COVID-19 and related policy limits their ways to communicate and obtain information and thus influence the physical and mental health development of teenagers. Therefore, problem behaviors are more likely to occur. On the one hand, the long-term sit around home will lead to more family conflicts, and might lead to problem behaviors. On the other hand, the family has become the main living environment for teenagers and the most important base for solution of problem behaviors. Therefore, the influence of family factors on adolescent problem behavior is more significant during the epidemic of COVID-19.

Among the family factors affecting adolescents' problem behavior, attachment is one of the important factors. Attachment is generally defined as the special emotional bonds between infants and their caregivers (generally mothers). It comes from the interaction between infants and their parents. Therefore, the initial researchers focused on how the relationship between mother and infant enriched and changed with the growth of infants. Now, researchers generally believe that attachment is an important aspect for human's adaptation, because it not only improves the possibility of infant survival, but also constructs the characteristics of lifelong adaptation, and helps individuals develop in the direction of a better adaptation[7]. Parental attachment is an important factor affecting problem behavior, and the change of parent-child relationship becomes more and more obvious in the process of getting along with parents. However, the relationship between parental attachment and problem behavior during the epidemic of COVID-19 is still unknown. Therefore, it is particularly important to study the relationship between attachment and problem behavior of adolescents during the epidemic to provide some implications for the intervention of problem behavior among adolescents.

Why parent-child attachment could affect teenagers' problem behavior? According to the theory of attachment control system model, long-term safe attachment can build personal resources and help individuals form positive coping [7]. From this perspective, the influence of parent-child attachment on problem behavior might via resilience. Resilience is a personal trait or ability that enables individuals to maintain relatively stable 
psychological and physical functions in the face of destructive events. From a process perspective, it can also be defined as a positive adaptation process in an adverse environment. Psychological resilience mainly includes two abilities: the ability to actively deal with, overcome, and adapt to traumatic experience; The ability to rebuild oneself, grow from trauma and move on[8]. There was a significant negative relationship between resilience and negative mental health variables such as depression and anxiety[9]. Therefore, the protective effect of resilience can prevent the injury caused by trauma. Based on the attachment theory, this study proposed that resilience can act as a mediating factor between parent-child attachment and problem behavior during the epidemic of COVID-19 among adolescents.

\section{METHOD}

\subsection{Participants}

The convenient sampling method was used to recruit participants. A total of 69 (19 males and 50 females) teenagers with an average age of 15.83 years old $(\mathrm{SD}=$ 1.06 years old) were recruited from three ordinary high schools in Jiangxi, Hebei and Guangdong province. All the questionnaires were collected by means of online.

\subsection{Procedure}

Participants were first arranged to fill the informed consent form first. Then the experimenter send the online link of questionnaires to participants, and participants fill these questionnaires online. The questionnaires include the Inventory of Parent Attachment, the Strength and Difficulty Questionnaire, the Adolescent Resilience Scale, and the questionnaire related to demographic variables. After filling these questionnaires, participants would receive 10yuan for their participation.

\subsection{Measures}

Parent-Child Attachment Questionnaire. Parent-child attachment was measured by the Inventory of Parent and Peer Attachment [10]. This self-report measure of attachment assesses children's continuum of secure attachment with their parents, as reported by the children. The scale includes the following two subscales that use a five-point scale ranging from 1 (never) to 5 (always): the mother-child attachment subscale (15 questions) and the father-child attachment subscale (15 questions). Each subscale have three dimensions: trust, communication, and alienation. A higher score indicated that the children were more securely attached. The Chinese version of this scale has good internal consistency (Ju et al. 2011; Wu and Wang 2014). For the father-child attachment subscale and the mother-child attachment subscale, the Cronbach's alpha values were .69 and .71 , respectively.

Strengths and Difficulties Questionnaire. The Strengths and Difficulties Questionnaire was used to access the problem behavior of adolescences[11]. This scale includes 25 items, and five dimensions, these dimensions includes prosocial behavior, emotional problems, hyperactivity-inattention, conduct problem, and peer problem. Each behavioral item is rated on a 3point Likert scale $(0=$ Not true, $1=$ Somewhat True, and $2=$ Certainly True). The Cronbach's alpha values were .79 in the current study.

Adolescent Resilience Questionnaire. The Adolescent Resilience Questionnaire was used to access the resilience of adolescence[12], it includes 27 items, these items include several aspects of resilience like introspection and reflection, social skills, optimism, problem solving, and so on. All the items were answered raging from 0 (totally disagree) to 6(totally agree). The Cronbach's alpha values were .91 in the current study.

\subsection{Data analysis}

The mediating model was estimated with parent attachment as the predictor, behavior problem as the outcome, and resilience as a mediator in the model. Direct and indirect effects were tested with the bootstrapping method of 1,000 samples, generating a bias-corrected percentile-based bootstrap with $95 \%$ confidence intervals. This method is a nonparametric resampling procedure which can overcome the problem of low power in traditional $\mathrm{z}$ statistics and does not need to assume multivariate normality of the sampling distribution [13]. Analyses were performed using the process plug-in in SPSS.

\section{RESULTS}

\subsection{Characteristics of behavior problem among adolescence during the COVID-19}

First, we compare the level of each kind of behavior problem, the results indicated the difference of the outbreak of each kind of behavior problem was significant $(\mathrm{F}(3,204)=23.33, \mathrm{p}<0.001)$. Post-hoc analysis indicated the number of hyperactivityinattention and emotional problems was significantly higher than conduct and peer problems (see Table 2.).

Table 2. Descriptive data of the level of each kind of behavior problems

\begin{tabular}{ccccc} 
& conduct & hyperactivity-inattention & peer & emotional \\
\hline M & 3.19 & 4.86 & 2.70 & 4.04 \\
\hline
\end{tabular}




\begin{tabular}{lllll}
\hline SD & 1.81 & 2.21 & 1.63 & 2.74 \\
\hline
\end{tabular}

Then we compared the level of behavior problems in gender, guardian, and whether live with parents. The results indicate there was no gender difference in each kind of behavior problem (ps $>.05)$. And there were no differences between each kinds of guardian ( $p s>.05$ ). Also, there were no differences between adolescences who live with parents and adolescences who did not (ps $>.05)$.

\subsection{Descriptive analysis and correlations between each variable}

The descriptive results and correlations between each variable were presented in Table 1 . The results indicate mother' $s$ trust significantly correlates with behavior problems and resilience. Both father and mother' $\mathrm{s}$ alienation significantly correlate with behavior problem and resilience. While father' $\mathrm{s}$ trust, father' $\mathrm{s}$ communication, and mother' $\mathrm{s}$ communication did not correlate with behavior problems. What's more, father' $\mathrm{s}$ trust and alienation significantly correlates with prosocial behavior.

Table 1 Descriptive results and correlations between each variable.

\begin{tabular}{|c|c|c|c|c|c|c|c|c|c|}
\hline & 1 & 2 & 3 & 4 & 5 & 6 & 7 & 8 & 9 \\
\hline \multicolumn{10}{|l|}{ 1.Ftrust } \\
\hline 2.Mtrust & $.59 * \star$ & & & & & & & & \\
\hline 3.Mcommunication & $.45^{\star \star}$ & $.76^{\star \star}$ & & & & & & & \\
\hline 4.Fcommunication & $.67^{\star \star}$ & $.46^{\star \star}$ & $.67^{\star \star}$ & & & & & & \\
\hline 5.Falienation & $-.50 * \star$ & $-.26^{\star}$ & $-.35^{\star \star}$ & $-.44^{\star \star}$ & & & & & \\
\hline 6.Malienation & $-.28^{\star}$ & $-.41^{\star \star}$ & $-.56^{\star \star}$ & $-.42^{\star \star}$ & $.70^{\star \star}$ & & & & \\
\hline 7.prosocial behavior & $.30 *$ & 0.24 & 0.23 & 0.19 & $-.24^{\star}$ & -.08 & & & \\
\hline 8.behavior problem & -.23 & $-.28^{\star}$ & -.17 & -.10 & $.31^{\star \star}$ & $.37^{\star \star}$ & -.04 & & \\
\hline 9.resilience & $.47^{\star \star}$ & $.52^{\star \star}$ & $.48^{* *}$ & $.39 * *$ & $-.44^{\star \star}$ & $-.48^{\star \star}$ & .19 & $-.69 * *$ & \\
\hline M & 15.93 & 16.35 & 14.00 & 12.14 & 15.64 & 16.01 & 6.48 & 14.78 & 84.35 \\
\hline SD & 4.42 & 4.16 & 5.38 & 5.10 & 3.99 & 3.87 & 2.04 & 6.41 & 16.26 \\
\hline
\end{tabular}

Note: Ftrust $=$ trust of father, Mtrust $=$ trust of mother, Mcommunication $=$ communication of mother, Fcommunication $=$ communication of father, Falienation $=$ alienation of father, Malienation $=$ alienation of mother.

\subsection{Relationship between parent attachment and behavior problem, resilience as a mediator.}

Last, we run a series of mediating analysis with each dimension of attachment as predictor, behavior problem as outcome variable, and resilience as mediator. The results indicated that resilience plays a mediating role between mother' $\mathrm{s}$ trust, father' $\mathrm{s}$ alienation, mother' $\mathrm{s}$ alienation, and behavior problem (see Figure 1.). More specifically, adolescences with the higher level of mother' s trust, have the higher level of resilience, and have lower level of behavioral problem. And adolescences with the higher level of mother' $\mathrm{s}$ alienation and father' $\mathrm{s}$ alienation, have the lower level of resilience, and have higher level of behavioral problem. 


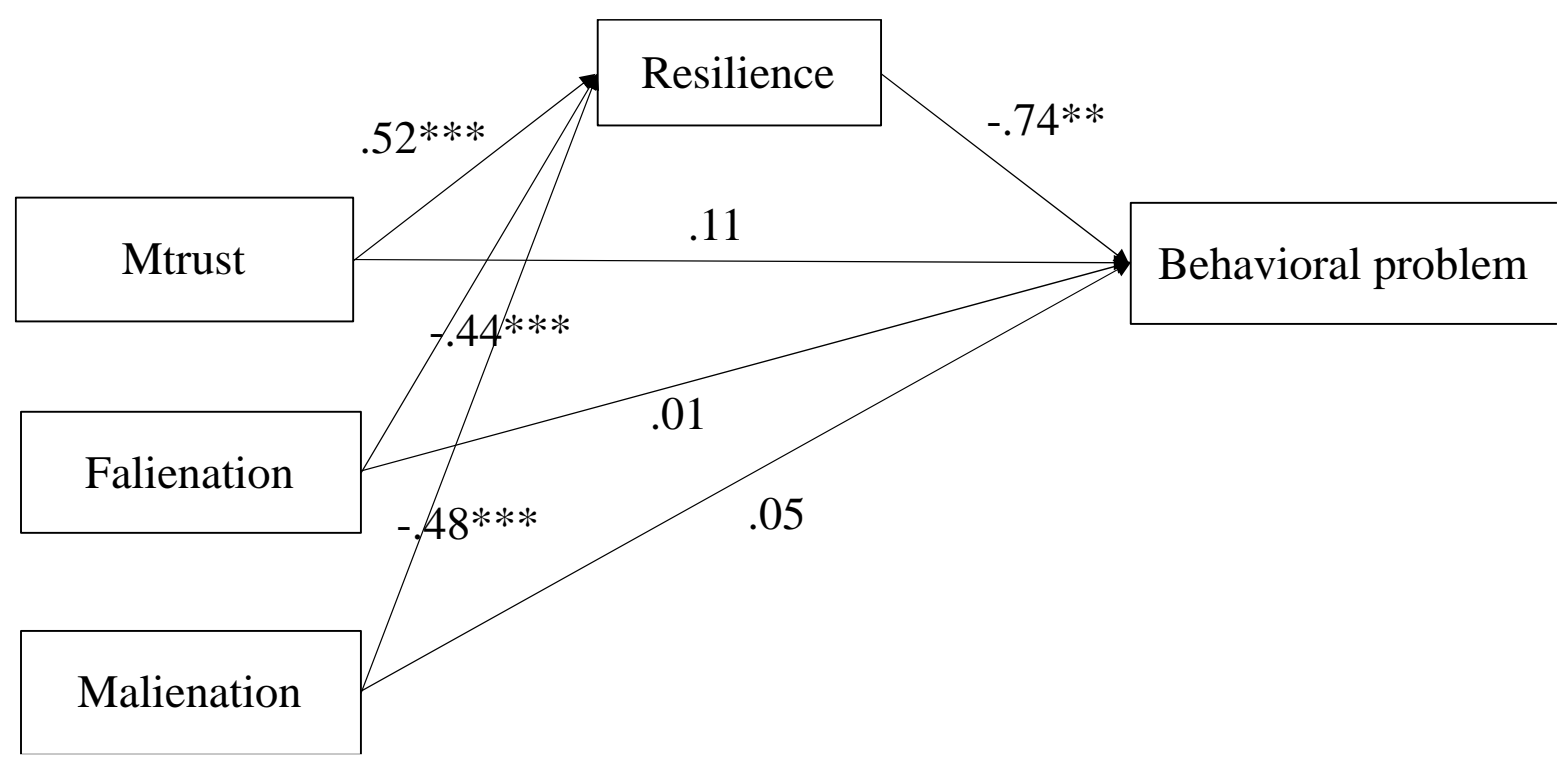

Note: Mtrust $=$ trust of mother, Falienation $=$ alienation of father, Malienation $=$ alienation of mother.

Figure 1. The mediating model of parent attachment, resilience, and behavioral problem.

\section{CONCLUSION}

The current study investigated the characteristics of the behavior problem of adolescences during the epidemic of COVID-19. We also investigate whether parent attachment could influence behavior problem via resilience. The results indicates that adolescents' behavior problems were prominent, among which hyperactivity-attention problems and emotional problems were significantly higher than other behavior problems during the COVID-19. The mediating analysis revealed that resilience plays a mediating role between mother's trust, father's alienation, mother's alienation, and behavior problem. These results provide some implications for learning the influence of COVID-19, and the intervention of behavior problem during the epidemic of COVID-19.

The descriptive results of the behavior problem of adolescents have some different characteristics with the result during the normal situations. The hyperactivityattention problems and emotional problems are higher than other behavior problems during the COVID-19. While previous research have found that level of different kinds of behavior problems almost identical among adolescents in normal situations[3]. This inconsistent results indicate that the outbreak of the COVID-19 actually influences the proportion of each kinds of behavior problem. During this epidemic time, adolescences have to stay more time at home, this situation hinder the expression of the conduct problem and peer problem, but amplified the chance to express the hyperactivity-attention problems and emotional problems.

The mediating analysis results indicates that mother's trust, father's alienation and mother's alienation could influence behavior problem via resilience, this results is consistent with previous research about the relationship of social support, resilience, and mental health, previous research indicates that social support is particularly important in maintaining good physical and psychological health in the presence of environmental risks, which could help individuals to develop resilience[14]. Pinkerton \&and Dolan (2007) proposed that family support and social capital could link coping by resilience. Some research within the organizational context has also found that psychological or emotional support could positively predict resilience, promote work performance, and improve occupational psychological health[16]. As parents act as important social support individuals for adolescences, parents' trust, especially mother's trust could help individuals build resilience and then influence behavior problem. While on the contrary, parents' alienation could harmful for the resilience and increase the number of behavior problems among adolescents. Our results also consistent with the control system model of attachment, this model indicates attachment could build personal resources and help individuals form positive coping. Our results provide some new evidence to this model.

The current research also proved some implications for the intervention of the behavior problem during the epidemic of COVID-19. Parents, teachers, or school could pay more attention to hyperactivity-attention problems and emotional problems. More importantly, they could provide children more trust and support to help them build resilience during their daily life. These will helpful for the mental health of adolescents during the public health emergency like the COVID-19.

\section{REFERENCES}

[1] L. W. Brinke, A. T. A. Menting, H. D. Schuiringa, J. Zeman, and M. Deković, "The structure of emotion 
regulation strategies in adolescence: Differential links to internalizing and externalizing problems," Soc. Dev., vol. 30, no. 2, pp. 536-553, 2021.

[2] J. E. Donovan and R. Jessor, "Structure of problem behavior in adolescence and young adulthood.," $J$. Consult. Clin. Psychol., vol. 53, no. 6, p. 890, 1985.

[3] S. Meng, Z. Chen, Q. Li, and Y. Wang, "Survey and analysis of problem behavior for middle school students," Educ. Sci., vol. 27, no. 4, pp. 43-48, 2011.

[4] D. V Ary, T. E. Duncan, A. Biglan, C. W. Metzler, J. W. Noell, and K. Smolkowski, "Development of adolescent problem behavior," J. Abnorm. Child Psychol., vol. 27, no. 2, pp. 141-150, 1999.

[5] C. Eppler, "Ecosystem in family systems theory," Encycl. Couple Fam. Ther., pp. 828-832, 2019.

[6] J. P. Hoffmann, "Family structure, community context, and adolescent problem behaviors," $J$. Youth Adolesc., vol. 35, no. 6, pp. 867-880, 2006.

[7] M. Mikulincer and P. R. Shaver, "A model of attachment-system functioning and dynamics in adulthood," in Attachment in adulthood: Structure, dynamics, and change, 2nd ed., M. Mikulincer and P. R. Shaver, Eds. New York: The Guilford Press, 2016, pp. 27-46.

[8] G. A. Bonanno, "Loss, trauma, and human resilience: Have we underestimated the human capacity to thrive after extremely adverse events?," Am. Psychol., vol. 59, no. 1, pp. 20-28, 2004.

[9] M. E. Beutel, H. Glaesmer, J. Wiltink, H. Marian, and E. Brähler, "Life satisfaction, anxiety, depression and resilience across the life span of men,"
Aging Male, vol. 13, no. 1, pp. 32-39, 2010.

[10] G. C. Armsden and M. T. Greenberg, "The inventory of parent and peer attachment: Individual differences and their relationship to psychological well-being in adolescence," J. Youth Adolesc., vol. 16, no. 5, pp. 427-454, 1987.

[11] R. Goodman, "The Strengths and Difficulties Questionnaire: a research note," J. child Psychol. psychiatry, vol. 38, no. 5, pp. 581-586, 1997.

[12] D. Gartland, L. Bond, C. A. Olsson, S. Buzwell, and S. M. Sawyer, "Development of a multidimensional measure of resilience in adolescents: the Adolescent Resilience Questionnaire," $B M C$ Med. Res. Methodol., vol. 11, no. 1, pp. 1-10, 2011.

[13] K. J. Preacher and A. F. Hayes, "Asymptotic and resampling strategies for assessing and comparing indirect effects in multiple mediator models," Behav. Res. Methods, vol. 40, no. 3, pp. 879-891, 2008.

[14] F. Ozbay, H. Fitterling, D. Charney, and S. Southwick, "Social support and resilience to stress across the life span: A neurobiologic framework," Curr. Psychiatry Rep., vol. 10, no. 4, pp. 304-310, 2008.

[15] J. Pinkerton and P. Dolan, "Family support, social capital, resilience and adolescent coping," Child Fam. Soc. Work, vol. 12, no. 3, pp. 219-228, 2007.

[16] M. Bernabe and J. M. Botia, "Resilience as a mediator in emotional social support's relationship with occupational psychology health in firefighters," J. Health Psychol., vol. 21, no. 8, pp. 1778-1786, 2016. 